\title{
Response to: Does Segmental Kyphosis Affect Surgical Outcome after a Posterior Decompressive Laminectomy in Multisegmental Cervical Spondylotic Myelopathy?
}

\author{
Akshay Jain, Tarush Rustagi, Gautam Prasad, Tushar Deore, Shekhar Y. Bhojraj \\ Spine Unit, Lilavati Hospital and Breach Candy Hospital, Mumbai, India
}

We sincerely thank you for your interest in our article. Here we provide the answers to your queries.

Queries 1, 2, and 3: The aim of surgery in cervical spondylotic myelopathy (CSM) is spinal cord decompression. As long as the cervical curvature has overall lordotic alignment (types C2 and C3 in our classification), posterior decompression is effective. The only drawback of this approach is the loss of cervical alignment at the long-term follow-up, which is more frequent in patients with preoperative kyphosis. Our study showed that this had no effect on the functional outcome. The proposed hypothesis is that a degenerated spine autostabilizes with time, thus preventing significant kyphosis. This study compares the functional outcome of spinal cord decompression on the basis of recovery rate. Although we did not include data on neck pain in this paper, we have compared it in both groups and there is no significant difference at the longterm follow-up. We propose that CSM with an overall lordotic cervical curvature should be decompressed only, irrespective of the patient's age.

Query 4: We did not separately study this subset of patients and therefore cannot comment on it.

Query 5: No patient in this series required revision surgery.

Query 6: It is wrongly mentioned. The correct sentence is "Long duration of symptoms before surgery and an age of $<57$ years positively correlated with the recovery rate. Furthermore, there was a positive correlation between a high preoperative japanese orthopaedic association score and the recovery rate.

\section{Conflict of Interest}

No potential conflict of interest relevant to this article was reported. 